Derecho y Realidad

Vol.13 - Núm. 26 • Julio-Diciembre de 2015

Págs. 235-264. ISSN:1692-3936

\title{
Acceso al derecho a la educación en la población femenina reclusa de Colombia*
}

\author{
Access to the right to education in female \\ inmates of Colombia
}

\author{
Jeimy Tatiana Rodríguez Naranjo**
}

\section{Resumen}

Esta investigación tiene como objeto establecer la importancia del acceso del derecho humano a la educación como pauta de resocialización dentro del cumplimiento de la pena de la población carcelaria femenina de Colombia. En el contexto en el que se estableció esta investigación, convergen diferentes factores sociales, económicos, educativos y culturales que afectan el goce al derecho a la libertad. Se realiza un análisis desde el ámbito jurídico de los derechos humanos (positivización) y el reconocimiento de la mujer reclusa como sujeto de derechos, enfocado en resaltar la importancia de un proceso adecuado de resocialización por medio del acceso a la educación, que dignifique a la población carcelaria en el goce de sus derechos humanos $(D H)$, pues debido a la pérdida de libertad, se ha limitado el goce de los derechos sociales y civiles, además de la caótica crisis de hacinamiento y

Fecha de aceptación: 15 de agosoto de 2015

Concepto de recepción: 14 de septiembre de 2015

Fecha de aprobación: 23 de octubre de 2015

Este artículo tiene demuestra la responsabilidad del estado colombiano no solo frente al derecho a la educación si no a la igualdad de género y sus condiciones para acceder a educación de calidad.

** Docente licenciada en Educación Básica. Especialista en Informática para la Docencia de la Universidad Pedagógica y Tecnológica de Colombia. Estudiante de Maestría en Derechos Humanos de la Universidad Pedagógica y Tecnológica de Colombia. tatysrod@hotmail.com. 
violencia intramuros. Se citan estudios sobre el estado del tema, tales como El acceso al derecho a la educación en contexto carcelario como pauta resocializadora, a partir de las investigaciones de Francisco José Scarfó (2002), Abraham Magendzo 2004, Katarina Tomasevski (2009), Marcela Lagarde (2002), Paul Freire (1965), además se citan la Declaración Universal de los Derechos Humanos, los pactos internacionales, la jurisprudencia nacional y las diferentes políticas públicas encaminadas al goce del derecho humano a la educación en estado de reclusión; y finalmente se establecen conclusiones pertinentes al tema de investigación.

\section{Palabras clave}

Educación, derecho, reclusión, derechos humanos, resocialización, dignidad.

\section{Abstract}

This research aims to establish the importance of the human right of access to education as a guideline of rehabilitation within the execution of the sentence of the female prison population of Colombia. In the context in which this research was established, reunite various social, economic, educational and cultural factors that affect the enjoyment of the right to freedom. An analysis is done from the legal field of human rights (positivization) and recognition of the female recluse as subjects of rights, aimed to highlight the importance of a proper process of rehabilitation through access to education that dignify the prison population in the use of their human rights, because the loss of freedom limits the exercise of social and civil rights and the chaotic crisis of overcrowding and inner prison violence. Many studies are cited and also the Universal Declaration of Human Rights, international agreements, national jurisprudence and various public policies on the use of the human right to education in prison; finally are cited relevant conclusions to this research.

\section{Keywords}

Education, law, detention, human rights, resocialization, dignity. 


\section{Sumario}

1. Introducción

2. Materiales y métodos

2.3 Mujer y cárcel

2.4 La pedagogía crítica como parte de la teoría crítica en la educación intramuros

2.5 La reincidencia efecto de la falta de pautas claras de reinserción

2.6 Sistema penitenciario en Colombia

2.7 Estructura educativa del Inpec

3. Resultados

3.1 Población reclusa en Colombia

3.2 Edad

3.3 Escolaridad

3.4 Acceso al derecho a la educación de la población reclusa interna

4. Discusión

5. Conclusiones

\section{Método}

Jurídico estadístico.

\section{Metodología}

Lo que se busca es determinar el nivel de acceso a la educación en Colombia para las mujeres reclusas. La realización de este estudio está basada en datos estadísticos que luego son analizados para determinar el índice de cumplimiento o vulneración de los derechos fundamentales de estas personas. ¿En realidad se ve materializado el objeto de la pena en el sistema penitenciario que se maneja en Colombia? 


\section{Introducción}

En la investigación que a continuación se presenta se enfatiza en determinar el nivel de acceso que tienen las reclusas colombianas al derecho fundamental de educación, resaltando su influencia en el proceso de resocialización. El estado colombiano al ser un estado de derecho debe ser garante del cumplimiento e inclusión de todos los nacionales en la consecución y goce efectivo de los derechos fundaméntales citando en esta investigación la población reclusa femenina en Colombia y su nivel de acceso al derecho a la educación, como derecho humano. Se sitúa la temática de población carcelaria en fijar a porcentajes de hacinamiento, de determinar cuál es el delito de mayor rango de incidencia, pero no se fijan que la reincidencia de delitos por parte de los reclusos, el informe estadístico del INPEC a junio de 2015 que presentó 170.437 reclusos de los cuales en sus diferentes modalidades de penalización la reincidencia corresponde a 14.095 personas. Asimismo, el 93,0 \% de los reincidentes son hombres y el restante 7,0\% mujeres esto es el reflejo de una inadecuada practica dentro del proceso de resocialización.

La vulneración del acceso al derecho a la educación de esta población en situación de detención los margina y limita del goce de sus derechos fundamentales. Este lugar actúa como un depósito de individuos, una alternativa sería como afirma el maestro especialista en educación para adultos y presidente del grupo de estudios sobre educación en cárceles en Argentina Francisco José Scarfó quien postula que en la cárcel la educación ejerce una fuerza de transformación:

La necesidad de garantizar el derecho a la educación a los detenidos es de vital importancia, no solo por ser un derecho, que hace a la esencia de todo ser humano, sino también por el beneficio personal de quien recibe educación y el impacto auspiciosos de una educación en Derechos Humanos en la participación y pertinencia real en la sociedad y en la construcción de la cultura en el marco de los Derechos Humanos. (Scarfó, 2002)

Es el Estado democrático el que debe diseñar un plan de educación a los sujetos en situación de detención, enfocado en la formación cognitiva, humana y laboral de personas adultas. Fuera de ser una simple capacitación, debe trascender el ser humano tratando de rescatar la dignidad perdida al limitar su libertad. El objetivo de la educación en contexto carcelario tiene un doble efecto positivo: ayuda al recluso a reconocer sus derechos y a respetar los de los demás. El Estado debe establecer un contenido normativo contextualizado en esta idea, en el que converjan como ejes generadores la vida humana, la integridad personal, la libertad, la igualdad, la tolerancia, la justicia y el desarrollo social. Lo anteriormente expuesto solo se alcanza con la educación de todos los ciudadanos, sin limitación alguna. El ser 
humano que se dignifica en el ejercicio y construcción como sujeto de derechos, participa activamente en la construcción social de su sociedad. Ante esta premisa, es Abraham Magendzo, docente chileno magíster en educación, quien acentúa sobre la educación en derechos humanos (EDH) la siguiente premisa:

La EDH tiene un rol fundamental en cuanto a hacer una contribución crítica a la prevención de violaciones de derechos humanos estimulando a las personas a participar de manera efectiva en la sociedad como miembros activos, informados, críticos y responsables. Desde esta perspectiva, la EDH debe ser considerada como una educación ética y política. Considera que el aprendizaje es una parte de la vida, antes que algo separado de otras partes de la vida e irrelevante para ellas. Está vinculada con los grandes problemas que sufre la sociedad, por ejemplo: pobreza crónica y desmoralizante; democracias frágiles e inestables; injusticia social: violencia; racismo; discriminación e intolerancia contra las mujeres, los homosexuales y las lesbianas; impunidad y corrupción. (Magendzo, 2002)

\section{Materiales y métodos}

\subsection{Estrategias de investigación}

El método investigativo se basa en el estudio descriptivo de enfoque cualitativo del estado de la cuestión en la interpretación de los instrumentos jurídicos universales, regionales y nacionales sobre el acceso al derecho a la educación de la población carcelaria de Colombia. $Y$ de enfoque cuantitativo cuando se investigan los datos estadísticos suministrados por el INPEC, que es el encargado de la administración del sistema penitenciario en Colombia. Además se hace una interpretación de los resultados a partir de un análisis comparativo con la situación de goce de los derechos humanos en la cárcel.

\subsection{Jurisprudencia internacional, regional y nacional sobre el derecho humano a la educación y la educación en el contexto carcelario}

En el contexto actual, en donde los planes de desarrollo y la legislación de cada Estado se enmarca en la globalización, es pertinente traer a relación el texto Contenido y vigencia del derecho a la educación, de Katarina Tomasevski (2002), relatora especial de las Naciones Unidas sobre el derecho a la educación en el periodo 1998-2004, quien resalta la vital importancia de la orientación actual de las estrategias de desarrollo mundial hacia la erradicación de la pobreza, convirtiendo el derecho a la educación en un instrumento poderoso para la incorporación de los derechos humanos. 
Un cínico podría discernir una disminución de la ambición en la transición del desarrollo a la erradicación de la pobreza, de la educación a la educación básica y de la educación como bien común a la educación como instrumento para la erradicación de la pobreza. La definición de la educación en la normativa internacional de los derechos humanos es por consiguiente una salvaguardia necesaria: abarca el derecho a la educación, los $\mathrm{DH}$ en la educación y la enseñanza de los DH. (Tomasevski, 2003, p. 11)

En afinidad con el postulado de Tomasevski, es claro afirmar que la educación es y será históricamente la herramienta de los Estados para insertar procesos de desarrollo en todos sus nacionales y procesos de ejercicio pleno de los derechos humanos.

Para citar la normatividad internacional desde la perspectiva universal en relación con el derecho a la educación como derecho humano, se citará la Declaración Universal de los Derechos Humanos (1948), haciendo énfasis en el artículo 26, que especifica el objeto central de esta investigación: "Toda persona tiene derecho a la educación", incluida allí la población carcelaria. Citamos también el Pacto Internacional sobre los Derechos Económicos, Sociales y Culturales, en cuyo artículo 13 se puntualizó "el derecho de toda persona a la educación", que deben garantizar todos los países miembros de este Pacto vigente en Colombia desde 1988.

También se referencia la Convención contra la Tortura y otros Tratos o Penas Crueles, Inhumanos o Degradantes, la cual fue adoptada por la Asamblea General de las Naciones Unidas el 10 de diciembre de 1984, debido a la situación de crisis carcelaria que viven los reclusos, donde las condiciones catastróficas sanitarias, alimenticias y de dormitorios, producto del hacinamiento, generan un contexto inhumano y que atenta contra la vida y la seguridad de las personas.

En el año 2009 fue presentando el Informe sobre el Derecho a la Educación de las Personas Privadas de Libertad, del relator especial de la ONU sobre el Derecho a la Educación, Vernor Muñoz, en el que afirma que el encarcelamiento no supone la renuncia a los derechos humanos y que las personas privadas de libertad constituyen uno de los grupos severamente marginados que están sometidos a la violación endémica de su derecho a la educación.

La Corte Interamericana de Derechos Humanos (CIDH) señala que varias organizaciones invocan las reglas mínimas de Naciones Unidas para el tratamiento de los reclusos, a fin de rescatar la dignidad y un tratamiento humano en los establecimientos penitenciarios de todos los países. Entre ellas podemos citar a nivel nacional de carácter gubernamental la Defensoría del Pueblo, Ia Procuraduría 
y la Corte constitucional, y de carácter no gubernamental la Fundación Caminos de Libertad.

En la normatividad del país, la Constitución Política establece el derecho a la libertad y las condiciones para la detención de las personas, prohíbe la tortura y los "tratos o penas crueles, inhumanos o degradantes".

En Colombia, en materia de educación en prisiones está señalado en el capítulo $\mathrm{V}$ Título III de la ley 115, Ley General de Educación.

El marco legal del Sistema Penitenciario está dado por la Ley 65 de 1993, por medio de la cual se expide el Código Penitenciario y Carcelario. Allí se incluye el tratamiento penitenciario en su artículo 94, el cual indica que la educación, al igual que el trabajo, constituye la base de la resocialización, el artículo 95 establece que la Dirección General del INPEC determina los estudios que se deben organizar en los centros penitenciarios y que sean válidos para la redención de pena; y en el artículo 98 se establece la enseñanza como actividad de redención, instrucción a otros en alfabetización, enseñanza primaria, secundaria, artesanal, técnica y de educación.

Entre la normatividad que se relaciona en el sistema penitenciario también está la jurisprudencia de la Corte Constitucional y los documentos Conpes que conciernen al sistema penitenciario y carcelario y al goce efectivo de los derechos humanos de la población carcelaria. La Corte Constitucional ha generado una serie de sentencias para determinar la forma de reivindicar algunos derechos humanos de los reclusos en contextos de dignidad (Corte Constitucional, sentencias T-153/98, 388/13, sentencia de Tutela 153 de 1998, M. P. Eduardo Cifuentes Muñoz).

Asimismo, hay varios documentos Conpes relacionados con el Sistema Penitenciario y Carcelario de Colombia, que confluyen en el mismo problema: dar solución a la situación de hacinamiento carcelario. Dentro de los documentos, señalaremos los siguientes:

Conpes 2797 de 1995 (DNP, 1995), se considera como el más detallado y profundo, porque diagnostica la situación carcelaria del país, visionando lo que serían los años venideros $y$, así mismo, formula recomendaciones para enfrentar los problemas existentes y los que se avecinan. La principal recomendación es la reiteración de construir nuevos centros carcelarios y la adecuación de otros, para enfrentar el grave problema de hacinamiento; además sugiere la "redistribución de la población reclusa para descongestionar", sin hacer especial énfasis en la distribución por género y diferencia de necesidades entre hombres y mujeres. Necesidades en infraestructura, ya que las mujeres tienen diferencias notables ante los hombres con respecto a compañía, intimidad, sexualidad, fisiología, como lo señalan Azóala 
y Yamacán en su texto "Mujeres Olvidadas" (2012), quienes postulan que las mujeres son una población especial en el entorno carcelario, tienen necesidades muy diferentes a los hombres y la mayoría de las cárceles fueron diseñadas pensando en los hombres, lo que dificulta su inclusión dando paso a la discriminación.

Conpes 3086 de 2000 (DNP, 2000), "Ampliación de la infraestructura penitenciaria y carcelaria". Enfoca su contenido en la inversión económica que se debe realizar para la construcción de once centros de reclusión que se hacían necesarios como medida para contrarrestar el hacinamiento que se proyectaba a 2004. Es pertinente recalcar la precisión que se hacía sobre la articulación que se debía realizar para trabajar sobre la oferta y demanda de la población carcelaria, dada la presión que sobre el Sistema Penitenciario y Carcelario ejerce la tendencia al aumento de la población reclusa y las limitaciones fiscales para igualar la oferta con la demanda carcelaria. Situación que hacía imprescindible que el Ministerio de Justicia y del Derecho liderara gestiones ante otras entidades estatales, dirigidas a buscar soluciones frente la problemática penitenciaria.

En el Conpes 3277 de 2004, por su parte, no se hace referencia a los temas de políticas criminal y penitenciaria, ni resocialización, respecto al Inpec, solo se menciona que hace parte del Sistema Nacional Penitenciario, al igual que los centros de reclusión (nacional, departamental y municipal), que en conjunto se denominan Establecimientos de Reclusión del Orden Nacional (ERON ). El tema de hacinamiento, por el contrario, es abordado de manera amplia y detallada en el documento.

Conpes 3575 de 2009, Estrategia para la Expansión de la Oferta Nacional de Cupos Penitenciarios y Carcelarios -seguimiento a los Conpes 3277 de marzo 2004 y 3412 de marzo de 2006 (DNP; 2004). En el documento se eluden los temas de políticas criminal y penitenciaria, y se sigue tratando lo pertinente al hacinamiento de los establecimientos carcelarios; también se recomienda lo relacionado con infraestructura y administración, sin citar por ejemplo lo pertinente a educación o capacitación.

Conpes 3828 de 19 de mayo de 2015. Según este documento, se fijarán las políticas públicas que enfocan el sistema penitenciario en Colombia, con el objetivo de fortalecer la capacidad institucional desde una perspectiva integral para garantizar la efectiva resocialización de la población privada de la libertad y el cumplimiento de los fines constitucionales de la pena en condiciones de dignidad humana. Este documento está no solo encaminado a la política penitenciaria y carcelaria, como hasta el momento se ha concentrado casi exclusivamente en la ampliación de la oferta de cupos, sino que pone sobre la mesa la participación de el Ministerio de Justicia y del Derecho, el Ministerio de Defensa Nacional, el Ministerio de Salud 
y Protección Social, el Ministerio del Trabajo, el Ministerio de Educación Nacional, el Ministerio de Tecnologías de la Información y las Comunicaciones y el Departamento Nacional de Planeación. Lo que reafirma la tesis expuesta en este capítulo, de considerar al ser humano como un ser íntegro y que debe vivir dignamente. Igualmente hace recomendaciones a los diferentes ministerios (Ministerio de Interior, Ministerio de Justicia y Derecho, Ministerio de Trabajo, Ministerio de Tecnologías, Ministerio de Defensa, Ministerio de Salud y Protección Social), al Departamento de Planeación, con acciones específicas que contribuyan al objetivo general del documento (resocialización a partir del acceso al derecho a la educación), pero falta aun la injerencia de la mujer específicamente en el diseño de estas políticas en cuanto al acceso al derecho a la educación, ya que no fija tareas concretas al Ministerio de Educación.

En síntesis, desde el ámbito universal hasta el nacional, se observa que la educación es un derecho de todas las personas, incluida la población carcelaria, y que, por lo tanto, sobre el goce al derecho a la educación giran ejes transformadores de la sociedad.

\subsection{Mujer y cárcel}

\subsubsection{Historia de las cárceles de mujeres}

Las instituciones de reclusión femeninas han tenido y tienen su propia historia, su propia filosofía, su propia lógica de funcionamiento y su propia fisonomía, porque a lo largo de los siglos ha habido una forma diferente de castigar a los hombres y mujeres que han vulnerado las leyes penales. El tipo de castigo para las mujeres transgresoras de las normas se ha ido desarrollando históricamente mediante la elaboración de un tipo de tratamiento y control que ha definido el sujeto de "mujer presa", como una mujer transgresora no solamente de las leyes penales -desviación delictiva- sino también de las normas sociales que regulan lo que ha de ser su condición femenina -desviación social-. Esta doble condición de mujer "desviada" ha conllevado severas discriminaciones en la forma de aplicar el castigo, que se han ido forjando y consolidando históricamente desde la aparición de las primeras instituciones de reclusión femeninas. Hoy estas prácticas institucionales de raíz religiosa persisten bajo formas más modernizadas en manos del Estado.

En la época de la Independencia se importan modelos penitenciarios franceses y españoles, con el objeto de contribuir al Estado-nación.

En la cárcel de mujeres, cuando el número de detenidas se aumentaba considerablemente con las de mala vida, solían enviarlas a los Llanos de San Martín o a las playas del Magdalena, en donde podían encontrar Ilaneros o bogas con quienes Ilevar vida marital. En 1890, las Hermanas del Buen Pastor Ilegan a Bogotá 
para establecer la primera casa de corrección de mujeres, en la cual dormían en buenas camas, tomaban sanos y abundantes alimentos en platos de loza de pedernal, se bañaban y jugaban con las hermanas.

En la reseña histórica del Inpec, se narra que el 14 de marzo de 1828, Bolívar ordenó la creación de presidios en las capitales de provincia, y diez años después se establecieron las casas de castigo. La orden bolivariana afirma que la privación de la libertad es un medio para asegurar la utilización del preso en trabajos penosos para el beneficio del Estado. La ley del 38 trae una clasificación que busca el distinto tratamiento de los delincuentes; los establecimientos y su régimen (especialmente el de trabajo) varían según la penal.

Los inicios de la Reclusión de Mujeres de Colombia data de 1893, que estaba ubicada en el barrio Las Aguas, año que por comisión del Gobierno nacional bajo el mandato de Carlos Holguín, encarga a las misioneras del Buen Pastor de la vigilancia y control de las mujeres a ellas encomendadas. La administración de la Reclusión estuvo a cargo de las Hermanas Misioneras del Buen Pastor, quienes mantuvieron la administración hasta los años 80 , donde pasó a manos de directores nombrados por la Dirección General de Prisiones (hoy Inpec). La rehabilitación se Ilevaba a cabo bajo los preceptos de una instrucción moral y religiosa, fortaleciendo, según las religiosas, la firmeza del espíritu.

Las nuevas instalaciones de la Reclusión de Mujeres "El Buen Pastor" inicia su construcción en el año de 1952, construcción que termina en 1957. El Gobierno del Frente Nacional es el que ordena en este año el traslado de las primeras internas a las nuevas instalaciones. Las residentes eran mujeres acusadas, en su mayoría, de ilícitos contra el patrimonio económico, la vida e integridad de las personas, producto inmediato de la conflictiva situación social del país de entonces.

\subsubsection{Género y punitividad}

Hay aspectos específicamente genéricos que hacen más opresivo el hecho carcelario para las mujeres. Entre otros, como sostiene la antropóloga feminista mexicana Marcela Lagarde en su texto Los cautiverios de las mujeres: madres, esposas, monjas, putas, presas y locas, la diferente significación de la prisión en la vida de hombres y mujeres. Aun cuando para ambos géneros la prisión tiene como consecuencia, además del castigo, el desarraigo y la separación de su mundo, para las mujeres es mucho mayor, ya que la mayoría son abandonadas por sus parientes en la cárcel. Ser delincuente y haber estado en prisión son también estigmas y culpas mayores para las mujeres. Las mujeres exconvictas quedan estigmatizadas como malas en un mundo que construye a las mujeres como entes del bien y cuya maldad es imperdonable e irreparable (Lagarde, 1993, p. 676). 


\subsubsection{Los derechos humanos de la mujer privada de la libertad}

Se analizan aspectos que afectan el goce efectivo de los derechos humanos de las reclusas con diversa causalidad, pero con relevancia en el hacinamiento, las graves deficiencias en el tratamiento carcelario, la ausencia de la familia, el goce de sus derechos sexuales y reproductivos (visita conyugal, intimidad, orientación sexual), (Defensoría del Pueblo, 2014).

A manera de conclusión de la categoría mujer y cárcel, podemos anotar que falta por parte del Inpec conocimiento de criterios claros para el reconocimiento de las necesidades propias y específicas de la reclusión para mujeres en los ámbitos de los derecho, en temas como: sanidad (baños unidades sanitarias), sexualidad, salud (ginecología, obstetricia), educación (talleres y aulas), familia (adecuar celdas madre y políticas de traslado y cumplimiento de pena) entre las más relevantes y urgentes de mejorar.

\subsubsection{Funciones de la educación en la cárcel}

Según lo dispuesto en las normas penitenciarias, particularmente en la Resolución 7302 de 2005, el enfoque de resocialización es de tipo ocupacional, esto quiere decir que se desarrolla a partir de actividades de trabajo, estudio y enseñanza, las cuales generan la posibilidad, cuando las normas penales sustanciales no lo restringen, de redimir pena. EI Inpec también cuenta con proyectos productivos. Según la información presentada por el Inpec (junio 2015), los establecimientos penitenciarios y carcelarios atienden a través de actividades ocupacionales a una población total de 90.096 individuos, los cuales representan el $74,5 \%$ de toda la Población Privada de Libertad (PPL).

La pertinencia y eficacia del acceso al derecho a la educación por parte de las reclusas logran el éxito cuando se analiza a la interna como un ser humano con necesidades, habilidades y destrezas no solo delictivas, sino que se establece su grado de escolaridad y el contexto en el que se ha desempeñado. Es urgente reivindicar el derecho a la educación de estos sujetos, ya que es en la educación donde converge la oportunidad y la realidad del desarrollo individual y social de este ser humano que ha sido vulnerado del goce efectivo de varios de sus derechos humanos, víctimas de una sociedad del silencio y la desigualdad. Pero la mujer, más allá de una protectora y trabajadora del hogar, es un sujeto con un pasado, capaz de construir un presente y proyectar un futuro. $\mathrm{Y}$ en la actualidad, la mujer gracias a la lucha de muchos años, tiene reconocimiento civil, democrático y ha podido acceder a esferas académicas, científicas, políticas y humanas que hace cincuenta años no veía, pero en las cárceles continua aún su relegación a minoría vulnerable, pues al ser el $7 \%$, no cuenta para el diseño e implementación de 
políticas públicas y medidas de protección, siendo los abusos sexuales, la imposibilidad de mantener su unidad familiar, la falta de oportunidades de trabajo y capacitación, los factores que disminuyen sus posibilidades de resocialización efectiva. Como las cárceles están diseñadas y visualizadas para los hombres, las necesidades que se desean suplir son de carácter masculino, vemos como los talleres de capacitación y las aulas están en las áreas de reclusión masculina y allí las mujeres no pueden ingresar, entonces se plantean actividades alternativas para las mujeres que no cumplen con las mismas características que las de los hombres.

\subsection{La pedagogía crítica como parte de la teoría crítica en la educación intramuros}

Se observó que la educación para adultos que se imparte en los ERON es pertinente, siempre y cuando tanto los actores como los currículos integren la Educación en Derechos Humanos, en los procesos académicos de educación básica, en la formación en competencias laborales y en competencias comunicativas, debido a que va a facilitar a la ciudadana exconvicta ejercer en libertad el goce de sus derechos al trabajo, a la seguridad social, a la familia, a la salud, a la educación superior o permanente, puesto que planeado ya su proyecto de vida, superará el efecto sicológico negativo que generó el acto delictivo, el encierro y el tiempo de condena. Esta premisa se valida con el texto de Paulo Freire:

(...) lo importante, desde el punto de vista de la educación liberadora y no "bancaria", es que, en cualquiera de los casos, las personas se sientan sujetos de su pensar, discutiendo su pensar, su propia visión del mundo, manifestada, implícita o explícitamente, en sus sugerencias y en la de sus compañeros. Porque esta visión de la educación parte de la convicción de que no puede ni siquiera presentar su programa, sino que debe buscarlo dialógicamente con el pueblo, y se inscribe, necesariamente, como una introducción a la pedagogía del oprimido, de cuya elaboración él debe participar. (Freire, 1965)

Pensar con Freire el acceso a la educación intramuros también ayuda en la paz, en el goce de la recreación a través de la lectura, en la elaboración o reelaboración del proyecto de vida de las reclusas, en el aprovechamiento productivo del tiempo, pero sobre todo a la reivindicación de su dignidad apoyada en el reconocimiento como sujeto de derechos.

Retomando el texto de Freire, la alfabetización es la oportunidad liberadora real:

La conciencia del analfabeto es una conciencia oprimida. Enseñarle a leer y escribir es algo más que darle un simple mecanismo de expresión. Se trata de procurar en él, concomitantemente, un proceso de concienciación, o sea, de 
liberación de su conciencia con vistas a su posterior integración en su realidad nacional, como sujeto de su historia y de la historia. (Freire, 1965, p. 6)

Cuando un ser educado es consciente de reconocerse como sujeto de derechos, su principal acción va a ser respetar y construir espacios de derechos humanos. Él se convertirá en un transformador de la sociedad y se proyectará desde lo personal hacia su dimensión social, que es el fin único de la educación, con una conciencia crítica política capaz de promover la acción social para superar estructuras opresivas (cárcel). La pedagogía de Freire está dirigida a los adultos oprimidos analfabetas contextualizándolos en esta investigación en la cárcel.

Podemos concluir que la resocialización se hace efectiva cuando el sistema penitenciario resume su funcionalidad en un esquema como el siguiente:

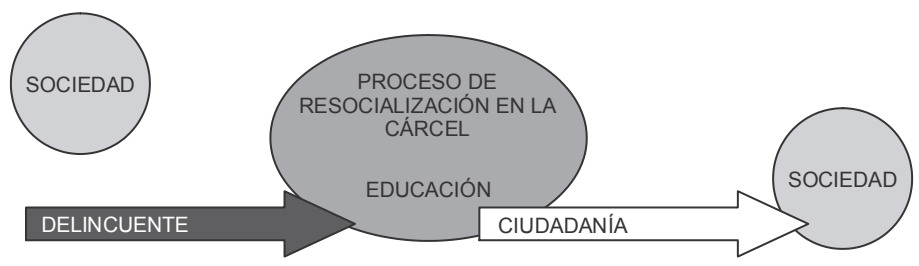

Figura 1. Resocialización efectiva

Observando esta figura analizaremos la situación previa al ingreso a la cárcel: se referencia a la persona excluida de la sociedad por razones económicas, sociales, políticas entre otras y se convierte en delincuente; luego ingresa en la cárcel a una reclusión que le permite acceder a la educación y sale de allí convertida en una ciudadana capaz de construir persona, familia y sociedad. Es pertinente aclarar que existen sujetos presos con características contrarias, pero lo que queremos es ratificar la importancia de cambiar su comportamiento penal para que puedan Ilegar a ser personas que ayuden en los procesos de transformación de la sociedad. Así lo vimos en la cárcel de Sogamoso, donde las reclusas que poseen estudios universitarios se convierten en monitoras de sus compañeras en la formación básica o las que poseen la capacitación en sastrería trabajan enseñando en el taller a sus otras compañeras, desempeñando dos actividades de redención de pena: trabajo y enseñanza.

\subsection{La reincidencia efecto de la falta de pautas claras de reinserción}

La afirmación que hace Ossa (2012) nos permite inferir que la reinserción se dimensiona desde varias ópticas, social, jurídica, educativa, penitenciaria, 
económica, pero se debe cuestionar sobre el deprimente estado de tener que volver a la cárcel a sufrir nuevamente la situación indigna de reclusión.

La reincidencia hace referencia a aquellos individuos que, habiendo sido condenados en ocasiones anteriores, han vuelto a ser privados de la libertad y se encuentran en condición de condenados por la comisión de nuevos delitos (Inpec, junio 2015)

\subsection{Sistema penitenciario en Colombia}

EI Inpec es la entidad encargada en Colombia de "administrar el Sistema Penitenciario y Carcelario, garantizando el cumplimiento de la pena privativa de la libertad, la detención precautelativa, la seguridad, la atención social y el tratamiento penitenciario de la población reclusa, en el marco de los derechos humanos". El Inpec es un establecimiento público adscrito al Ministerio del Interior y de Justicia, creado en 1992 mediante el decreto 2160 del mismo año. Por su carácter descentralizado funciona administrativamente con una sede ubicada en la ciudad de Bogotá y seis regionales (central, occidente, norte, oriental, noroeste y viejo Caldas) a cargo de las cuales se encuentran 139 establecimientos carcelarios en todo el país, clasificados por el Código de la siguiente manera:

\begin{tabular}{|c|c|c|c|c|c|c|}
\hline \multirow{2}{*}{ TOTAL } & \multirow{2}{*}{ DENOMINACIÓN } & \multicolumn{5}{|c|}{\begin{tabular}{|c|c|} 
& RESUMEN POR REGIONAL \\
\end{tabular}} \\
\hline & & CENTRAL & OCCIDENTAL & NORTE & NOROESTE & VIEJO CALDAS \\
\hline 1 & C.A.MI.S. E.R.E. & 1 & \begin{tabular}{|l|}
0 \\
\end{tabular} & 0 & \begin{tabular}{|l|l|}
0 \\
\end{tabular} & $\begin{array}{l}0 \\
0\end{array}$ \\
\hline 91 & E.P.M.S.C. & 24 & 18 & 8 & 17 & 15 \\
\hline 3 & E.P.M.S. & 2 & & & & \\
\hline 4 & E.P.M.S.C. - R.M. & 3 & 1 & & & \\
\hline 4 & E.P.M.S.C. -E.R.E. & & 1 & 2 & & 1 \\
\hline 1 & $\begin{array}{l}\text { E.P.M.S.C. } \\
\text { J.P. -E.R.E. }\end{array}$ & & & & & \\
\hline 1 & E.P.M.S.C. - J.P. & & & 1 & & \\
\hline 1 & E.P.M.S.C. - C.M.S. & 1 & & & & \\
\hline 2 & J.P. & 2 & 0 & & & \\
\hline 3 & E.P.A.M.S.-C.A.S. & 1 & & 1 & & \\
\hline 1 & EP & & 1 & & & \\
\hline 1 & $\begin{array}{l}\text { E.P.A.S.C.A.S - E.R.E. } \\
J P \text {. }\end{array}$ & & 0 & & 1 & \\
\hline 1 & $\begin{array}{l}\text { E.P.A.M.S. C.A.S. } \\
\text { E.R.E. }\end{array}$ & & 1 & & & \\
\hline 1 & $\begin{array}{l}\text { E.P.A.M.S - P.C. } \\
\text { E.R.E. }\end{array}$ & & & & & 1 \\
\hline 3 & E.P.C. & 2 & & 1 & & \\
\hline 3 & E.P. & 2 & & & 1 & \\
\hline 1 & E.C. & & & & 1 & \\
\hline 1 & E.C. - E.R.E. & & & 1 & & \\
\hline 1 & E.C. -P.S.M. & 1 & & & & \\
\hline 1 & E.C. - J.P. & & & 1 & & \\
\hline 1 & E.R.E. & & & 1 & & \\
\hline 5 & R.M. & & 1 & & & 3 \\
\hline 1 & R.M. - P.A.S. ERE & 1 & & & & \\
\hline
\end{tabular}

Figura 2. Establecimientos carcelarios administrados por el Inpec.

Fuente: Inpec (2015)

La estructura del INPEC está representada en el siguiente organigrama: 


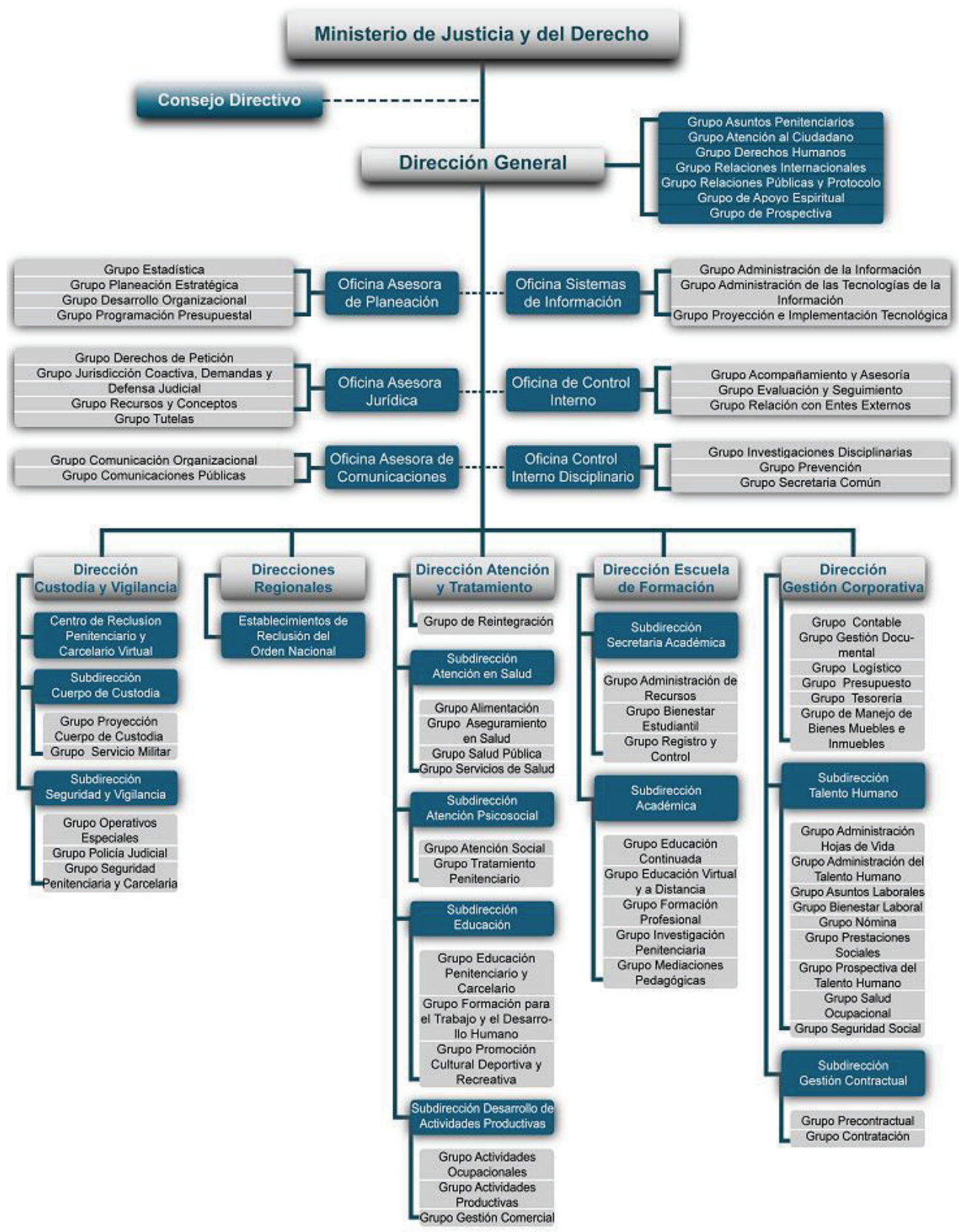

Figura 3. Organigrama Inpec.

Fuente: Decreto 4151 de 2011. 


\subsection{Educación penitenciaria a cargo del Inpec}

Es importante anotar que a partir de la expedición del Código Penitenciario y Carcelario se toma el concepto de tratamiento penitenciario como parte fundamental para alcanzar la resocialización.

EI Tratamiento Penitenciario tiene la finalidad de alcanzar la resocialización del infractor de la ley penal, mediante el examen de su personalidad y a través de la disciplina, el trabajo, el estudio, la formación espiritual, la cultura, el deporte y la recreación, bajo un espíritu humano y solidario.

Retomando la Ley 65 de 1993, en el artículo 95 se establece que la Dirección General del Inpec determina los estudios que se deben organizar en los centros penitenciarios y que sean válidos para la redención de pena; en el artículo 97 habla sobre redención de penas por estudio. $\mathrm{Y}$ establece:

A los detenidos y a los condenados se les abonará un día de reclusión por dos días de estudio. Se computará como un día de estudio la dedicación a esta actividad durante seis horas, así sea en días diferentes. Para estos casos no se podrán computar más de seis horas diarias de estudio.

En su artículo 98 contempla la enseñanza como actividad de redención, instrucción a otros en alfabetización, enseñanza primaria, secundaria, artesanal, técnica y de educación.

Asimismo, en el Acuerdo 0011de 1995 (31 de octubre), 'Por el cual se expide el Reglamento General al cual se sujetarán los reglamentos internos de los Establecimientos Penitenciarios y Carcelarios", en el capítulo noveno, referente a trabajo, estudio y enseñanza, establece: "Disposiciones relativas a redención de pena por trabajo, estudio o enseñanza. El trabajo, estudio o enseñanza en los centros de reclusión se regirá por lo dispuesto en las Resoluciones 3272 y 6541 de 1995, expedidas por la Dirección General del INPEC."

Un ente regulador de la realización de actividades dentro del establecimiento es la Junta de Evaluación de Trabajo, Estudio y Enseñanza. Este cuerpo colegiado aparece en el Acuerdo 0011de 1995 en su Título V Órganos penitenciarios y carcelarios, capítulo primero Órganos colegiados. En cada centro de reclusión funcionará una Junta de Evaluación de Trabajo, Estudio y Enseñanza encargada de conceptuar sobre el ingreso de los internos a las actividades laborales o educativas, de acuerdo con su aptitud y vocación, la disponibilidad del establecimiento y las actividades generadoras de redención, señaladas por la Dirección General del Inpec. Así mismo, 
controlará y evaluará en cada caso los trabajos realizados por los internos, la calidad, intensidad y superación por exámenes del estudio y la enseñanza. Esta junta está conformada por el director o subdirector, el coordinador del área educativa, el comandante de vigilancia y el jurídico.

En síntesis, el enfoque del sistema penitenciario colombiano confluye en el acceso a educación como pauta resocializadora a cargo del Inpec, siguiendo siempre en observancia de la dignidad y la igualdad.

\subsection{Estructura educativa del Inpec}

\subsubsection{Lineamientos Inpec-Ministerio de Interior y de Justicia}

Los lineamientos y recomendaciones que vienen a continuación se pueden leer como la conclusión del recorrido que hasta aquí se ha realizado. Se ordenan de acuerdo con la perspectiva de políticas públicas $y$, por tanto, se dividen en tres dimensiones: en relación con la formulación, con la ejecución y, finalmente, con la evaluación y seguimiento de la Política Penitenciaria en nuestro país. En cada una de estas dimensiones se han agrupado un conjunto de lineamientos y recomendaciones a partir de una formulación general, seguida de su respectivo desarrollo.

\subsubsection{Formulación de la política penitenciaria}

El tratamiento penitenciario debe ser entendido desde un punto de vista integral, lo que hace necesario considerar que la resocialización, como principal función de la pena en el momento de la ejecución penitenciaria, no se satisface solo con la existencia de actividades de trabajo, estudio y enseñanza dentro de los programas institucionales, sino que estas deben apuntar a crear herramientas para la socialización de los condenados y su efectivo regreso e incorporación a sus redes sociales y familiares con posterioridad a la ejecución de la pena.

En este sentido, los actuales programas transversales, que se articulan con los procesos de resocialización en los establecimientos penitenciarios, deben procurar no solo abrirse a los distintos miembros que conforman las redes de apoyo del condenado, como sus familias, sino que también deben generar estrategias para un mayor acceso a los mismos por parte de la población penitenciaria.

La "resocialización en serio" es el principal sentido que puede tener un sistema de sanciones penales en un Estado social de derecho. Con esto, y tal como ya lo ha mencionado la Organización de Naciones Unidas, la resocialización debe, por lo menos, comprenderse como una posibilidad para desarrollar alternativas para el ejercicio efectivo de los derechos que las personas privadas de la libertad previamente 
no habían podido ejercer; asimismo, tomar en serio la resocialización implica reducir al mínimo los efectos negativos del encarcelamiento, de tal manera que la vida en prisión no se convierta en la preparación de una futura carrera delictiva.

La mejor estrategia para enmarcar el tratamiento penitenciario en la protección y garantía de los derechos humanos, debe ser el reconocimiento y la aplicación de los estándares que la comunidad internacional ha desarrollado alrededor del tema. En tal sentido, el amplio catálogo de estándares internacionales categorizado como de derecho blando debe ser incorporado en la formulación de la política y en las interpretaciones necesarias en el marco de la ejecución de la misma. Las Reglas Mínimas y demás herramientas jurídicas que se mencionaron anteriormente, deben incorporarse como una base sobre la cual el Estado ejecuta el tratamiento penitenciario de cara a la preparación del retorno a sus redes sociales de los ciudadanos condenados (domiciliario, vigilancia electrónica, servicio social y laboral, judicial).

Articulado con lo anterior, para potenciar la función resocializadora de la pena, es necesario que no solo se reflexione sobre cómo estructurarse el proceso de tratamiento penitenciario, sino también sobre las alternativas a la prisión que permitan purgar sanciones y cumplir con un proceso restaurativo y de reintegración. Resulta menester, en consonancia con lo dispuesto por la Comisión Asesora de Política Criminal, proponer un proyecto de alternatividad penal y de alternatividad al derecho penal, que permita enfrentar ciertos problemas sociales más allá de los márgenes del sistema penitenciario.

\subsubsection{Finalidad de la pena hacia la reinserción social}

Como finalidad de la pena está el proceso de reinserción de los reclusos. Estos, antes de ingresar al penal, son personas que a las que se han vulnerado sus derechos fundamentales por sus condiciones sociales y económicas. De ahí la importancia de lograr un proceso de reinserción efectiva, en la que ellos puedan desarrollar sus capacidades laborales y personales, de tal forma que disminuya la probabilidad de la reincidencia delincuencial.

\subsubsection{Actores}

En el ámbito educativo carcelario convergen los siguientes actores:

- Los internos

- Los docentes y otros educadores

- El personal penitenciario de vigilancia

- Grupos civiles y religiosos 
En Ias penitenciarías y cárceles de Distrito Judicial habrá centros educativos para el desarrollo de programas de educación permanentes, como medio de instrucción o tratamiento penitenciario, que podrán ir desde la alfabetización hasta programas de instrucción superior. La educación impartida deberá tener en cuenta los métodos pedagógicos propios del Sistema Penitenciario, el cual enseñará y afirmará en el interno, el conocimiento y respeto de los valores humanos, de las instituciones públicas y sociales, de las leyes y normas de convivencia ciudadana y el desarrollo de su sentido moral. En esta línea de aprendizaje volvemos a citar a Scarfó (2012) y su línea pedagógica de enseñanza y aprendizaje de los derechos humanos como pauta de resocialización de la población carcelaria.

Las instituciones de educación superior de carácter oficial prestan un apoyo especial y celebran convenios con las penitenciarías y cárceles de Distrito Judicial, para que los centros educativos se conviertan en Centro Regional de Educación Superior Abierta y a Distancia (Cread), ahora Facultades de Estudios a Distancia, con el fin de ofrecer programas, previa autorización del Icfes. Estos programas conducirán al otorgamiento de títulos de educación superior.

Los internos analfabetos asistirán obligatoriamente a las horas de instrucción, organizadas para este fin.

La actividad educativa es objeto de redención de pena (así como la actividad laboral y de enseñanza), previa presentación de los cómputos del tiempo dedicado a esta labor y aprobación por parte del respectivo juez de ejecución de penas.

Los programas educativos dirigidos a la población reclusa se realizan siguiendo los lineamientos de la Ley 115 de 1994, Ley General de Educación y específicamente el Decreto 3011 de diciembre 19 de 1997, el cual establece las normas de la educación de adultos. Los programas se ajustan por sus principios básicos a las condiciones y necesidades particulares del sistema penitenciario y carcelario.

Se tiene en cuenta la tendencia universal en relación con la finalidad de la pena y el desarrollo de actividades educativas y laborales en prisión como un medio para generar opciones de vida a categorías que, traducidas en la realidad colombiana, a la luz de los datos antes expuestos, incluye sin duda a las mujeres.

Ahora bien, como señala Azóala \& Yamacán,

para entender el problema de la educación de las reclusas, resulta indispensable la conciencia y la aceptación de que las mujeres presas pertenecen a un grupo específico de nuestra estratificación social. [...] Antes de convertirse en reclusas, estas mujeres no habían podido ocuparse más que de su supervivencia por lo 
que es absurdo ofrecerles dentro del reclusorio una educación tradicional como única opción si ésta no ha tenido éxito en su vida en libertad. No es extraño, entonces, la alta deserción escolar dentro del penal, que no hace sino repetir y por razones semejantes, la que ya antes tuvieron fuera del penal. (2004)

La cobertura en los programas del área de educación para las mujeres recluidas, que son los mismos que se ofrecen a los hombres privados de libertad, es, de acuerdo con los datos proporcionados por la Dirección General del Inpec, la siguiente:

Tabla 1. Programas de cobertura

\begin{tabular}{|c|}
\hline Alfabetización \\
\hline Educación formal \\
\hline Educación no formal \\
\hline Educación informal \\
\hline Actividades culturales \\
\hline Actividades deportivas y recreativas \\
\hline Fuente: Inpec (2015)
\end{tabular}

\subsubsection{Programas educativos en los establecimientos carcelarios}

En los establecimientos carcelarios hay dos tipos de escolarización, la primera está dirigida por los colegios penitenciarios dirigidos por el Inpec, y la segunda está organizada por las secretarías de educación que focalizan operadores de educación para adultos. Los programas educativos orientados a la población reclusa se realizan siguiendo los lineamientos de la Ley 115 de 1994, Ley General de Educación y específicamente el Decreto 3011 de diciembre 19 de 1997, el cual establece las normas de la educación de adultos. Los programas se ajustan por sus principios básicos a las condiciones y necesidades particulares del sistema penitenciario y carcelario.

El modelo pedagógico del INPEC referencia los siguientes ciclos:

Tabla 2. Educación formal

\begin{tabular}{|c|c|}
\hline CICLO & GRADOS \\
\hline Ciclo 1 & 1 a 3 Básica primaria \\
\hline Ciclo 2 & 4 y 5 Básica primaria \\
\hline Ciclo 3 & 6 y 7 Básica media \\
\hline Ciclo 4 & 8 y 9 Básica media \\
\hline Ciclo 5 & 10 Media vocacional \\
\hline Ciclo 6 & 11 Media vocacional \\
\hline
\end{tabular}


Además de este programa cíclico, también hay programa de validación del bachillerato, programa de presentación de pruebas del lcfes, programa de educación superior.

\subsubsection{Programa de validación de estudios ICFES}

La validación es el procedimiento por medio del cual una persona demuestra que ha alcanzado el dominio de los conocimientos, habilidades y destrezas en las asignaturas y áreas de los grados o niveles de educación básica primaria, básica secundaria y media vocacional sin necesidad de probar su asistencia a clases regulares.

Así lo establecen los artículos 1 y 2el Decreto 2225 del 5 de noviembre de 1993, expedido por el Ministerio de Educación Nacional. La validación de estudios se realiza a través del Icfes, y es una alternativa educativa para la población reclusa, particularmente en aquellos establecimientos de reclusión donde no hay condiciones para que funcione un centro educativo. La Ley General de Educación (Ley 115 de 1994) la define en el marco de la educación no formal, como "la que se ofrece con el objeto de complementar, actualizar, suplir conocimientos y formar en aspectos académicos o laborales sin sujeción al sistema de niveles y grados".

\subsubsection{Programa de educación superior}

El Programa de Educación Superior hace parte de los programas de educación formal y se desarrolla en el marco de la educación abierta y a distancia. La Resolución 4105 de septiembre 25 de 1997 (por medio de la cual se expiden las pautas de educación penitenciaria) define la Educación a Distancia como "una alternativa innovadora respecto del modelo tradicional de educación, atendiendo a los requerimientos de permanente formación y perfeccionamiento profesional que demanda el mundo actual". Se adopta esta metodología por cuanto responde a las condiciones de seguridad del centro carcelario ya que no es necesario desplazar al interno a la institución educativa donde esté matriculado.

Las carreras que estudian: Administración de empresas, Administración de sistemas, Informática, Administración Ambiental, Administración de negocios, Administración agropecuaria, Administración de construcciones, Tecnología en administración comercial y financiera, Tecnología producción agropecuaria, Tecnología en electrónica, Tecnología en administración financiera y bancaria, Tecnología gestión comercial y de negocios, Tecnología gestión ejecutiva, Tecnología en obras civiles, Educación preescolar, Licenciatura en educación básica, Licenciatura en filosofía e historia, Licenciado en educación popular, Comunicación social, Salud ocupacional, Psicología social y comunitaria e Ingeniería de sistemas. 


\subsubsection{Infraestructura educativa en los establecimientos carcelarios}

De acuerdo con los datos proporcionados por la División Social del Inpec, hay treinta establecimientos que cuentan con Centros Educativos conformados como tales, esto equivale al $21.58 \%$ del total de establecimientos con Centros Educativos. En lo relacionado con la existencia de bibliotecas, hay aproximadamente cuarenta establecimientos que cuentan con áreas destinadas al préstamo de libros y de lectura, aunque la mayoría de los establecimientos tienen aunque sea de manera incipiente un esbozo de biblioteca, con ejemplares desactualizados, deteriorados o incompletos, y los espacios son generalmente bastante reducidos (repasemos lo anotado acerca de la infraestructura carcelaria). Las bibliotecas casi siempre se nutren de las donaciones logradas por campañas de recolección de libros que llevan a cabo los funcionarios encargados de las áreas educativas orientadas a tocar la sensibilidad de los estudiantes de colegios o de particulares.

\section{Resultados}

El análisis sobre el acceso al derecho a la educación en la población femenina reclusa de Colombia, se registra así:

\subsection{Población reclusa en Colombia}

Al finalizar el mes de junio, en Colombia se registraban 170.437 personas privadas de la libertad. A cargo del Inpec 165.860 (97,3 \%) y las restantes $4.577(2,7 \%)$ bajo la custodia y responsabilidad de los entes territoriales, Comandos de Fuerza y Dirección General de la Policía Nacional.

De la población penitenciaria y carcelaria a cargo del Inpec, $120.905(72,9 \%)$ se encontraba dentro de Ios ERON, 40.798 (24,6\%) en domiciliaria y $4.157(2,5 \%)$ con control y vigilancia electrónica.

Al finalizar este mes, la población carcelaria y penitenciaria intramuros estaba conformada por $112.423(93,0 \%)$ hombres y $8.482(7,0 \%)$ mujeres, para un total de 120.905 personas. La proporción de mujeres reclusas es de 1 a 13 con respecto a los hombres.

\subsection{Edad}

La población reclusa est discriminada en cuatro grandes rangos etarios. El guarismo más alto se ubica en la categoría de 30 a 54 años con 64.850 (53,6 \%), en segundo lugar quienes tienen entre 18 y 29 años, 48.303 (40,0\%). Las personas entre 55 y 64 años ocupan el tercer renglón con $5.711(4,7 \%)$ y finalmente, $2.041(1,7 \%)$ 
personas mayores de 65 años. En cada una de estas categorías, los hombres superan el $92 \%$ de participación con respecto a las mujeres.

Tabla 3. Edad población femenina reclusa

\begin{tabular}{|c|c|}
\hline \multicolumn{2}{|c|}{ Edad reclusas de Colombia } \\
\hline 18-29 años & 3.107 \\
\hline 30-54 años & 4.842 \\
\hline 55-64 años & 446 \\
\hline mayor de 65 & 87 \\
\hline
\end{tabular}

Fuente: Inpec

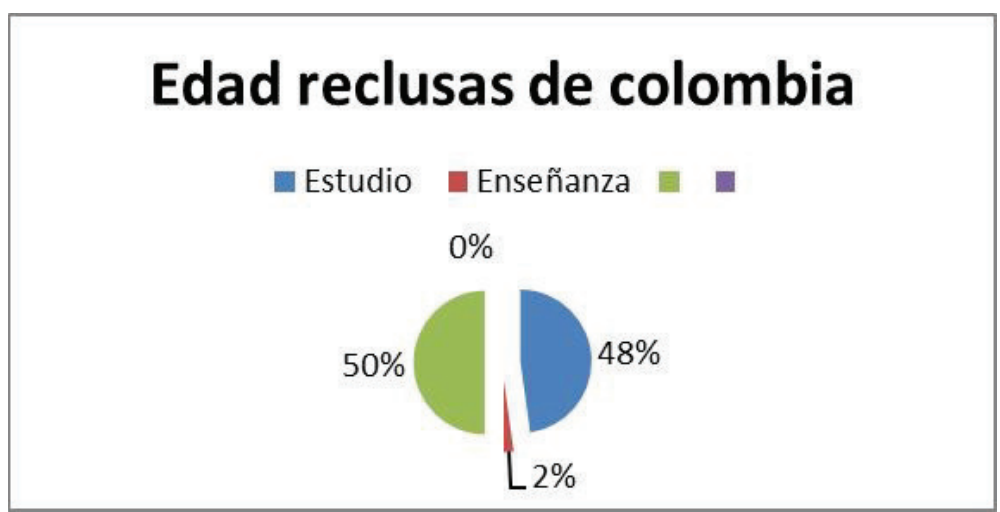

Figura 4. Edades reclusas Colombia.

Fuente: Tabla 3.

\subsection{Escolaridad}

EI perfil académico de las personas privadas de la libertad al momento de ingresar a los ERON correspondía a: 6.550 (5,4 \%) iletrados(as), 44.557 (36,9\%) con básica primaria (Ciclo 1, 16.876 y Ciclo 2, 26968)

Con respecto a quienes cursaron la básica secundaria y media vocacional, la cifra de internos corresponde a $65.803(54,4 \%)$ agrupado(as) en los ciclos 3 a 6 , de esta categoría.

SóOlo 20.937 internos(as) tienen el bachillerato completo.

De otra parte, $3.747(3,1 \%)$ tiene formación profesional en las diversas modalidades de técnico, tecnólogo y pregrado universitario. Únicamente $248(0,2 \%)$ reclusos(as) cuentan con especialización en un área profesional. 
Tabla 4. Nivel de escolaridad de las reclusas.

\begin{tabular}{|l|r|}
\hline Iletradas & 458 \\
\hline Básica primaria (ciclo 1 ciclo 2) & 2.119 \\
\hline Básica secundaria y media vocacional (ciclo 3 al 6) & 4.160 \\
\hline Bachillerato completo & 1.465 \\
\hline profesional & 263 \\
\hline especialistas & 17 \\
\hline Total & 8.482 \\
\hline
\end{tabular}

Fuente: Inpec

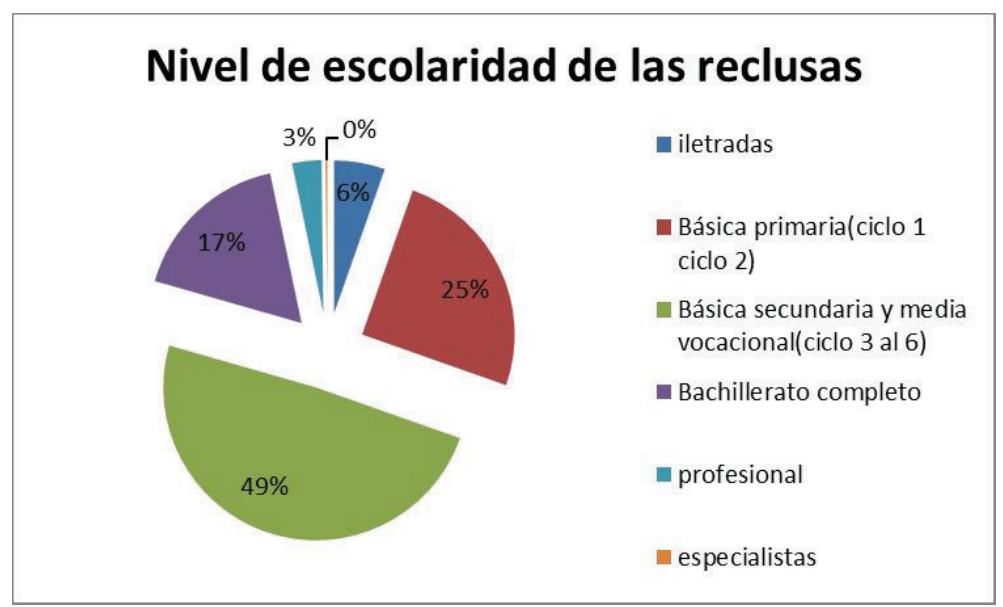

Figura 5. Nivel de escolaridad de las reclusas.

Fuente: Tabla 4.

\subsection{Acceso al derecho a la educación de la población reclusa interna}

Al terminar el mes en observación, el 74,5 \% (90.096) de la población interna en establecimientos de reclusión, participó en las tres grandes modalidades de ocupación: el 47,9 \% (43.152) realizó trabajos en las áreas industrial, artesanal, agropecuaria y de servicios administrativos (dentro de los establecimientos de reclusión), teniendo en cuenta las exenciones establecidas en la Ley. El 50,2 \% (45.226) asistió a los programas educativos, construyendo el fundamento para su resocialización, y por último, el 1,9 \% (1.771) de los internos(as) se desempeñó como instructor dentro del establecimiento.

Se analiza del acceso a la educación, que solo 162 mujeres participaron en programas de enseñanza y 3.497 en estudio, en total son 3.659, lo que corresponde solamente al $43 \%$ de la población total que accede a menos de la mitad. 
Tabla 5. Acceso educativo reclusas junio de 2015

\begin{tabular}{|c|c|}
\hline Estudio & 3.497 \\
\hline Enseñanza & 162 \\
\hline TOTAL & 3.659 \\
\hline
\end{tabular}

Fuente: Inpec

\section{Acceso al derecho a la educación}

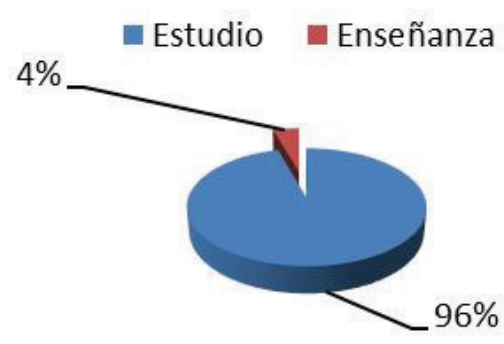

Figura 6. Acceso educativo reclusas junio de 2015.

Fuente: Inpec

\section{Discusión}

De acuerdo con el análisis estadístico, se reitera la tesis sobre la carencia de oportunidad para acceder al derecho humano a la educación de la población reclusa interna, debido a que menos de la mitad (43\%) goza de este derecho, como parte de su proceso de resocialización, y se concluye que esta es una causa de la reincidencia delictiva. Ante la privación de la libertad de los ciudadanos, debe haber suficientes alternativas al encarcelamiento, tales como pena pedagógica, pena comunitaria, pena constructiva.

En el estudio del acceso al derecho a la educación de la población carcelaria en Colombia se establecen aspectos positivos y negativos.

Se determina la factibilidad de la siguiente propuesta metodológica en las categorías teóricas expuestas, así:

- Derecho a la educación como derecho humano: la propuesta es deconstruir sobre lo que está implementado. Es pertinente continuar con la metodología en 
cuanto a educación básica y formal, ya que permite el acceso a un tipo de educación estandarizada con los programas educativos de las personas en libertad, generando equidad. Es importante continuar con la formación para el trabajo, pero se hace necesario equipar al sujeto de algo más que de conocimiento, equiparlo de competencias comunicativas y humanizarlo desde el ámbito de derechos humanos propios y ajenos.

Como lo afirma Magendzo (2002), educarlo en derechos humanos es proporcionarle la herramienta para trascender a la construcción de una sociedad pacífica, justa e integradora.

- Mujer y cárcel: generar políticas públicas que proporcionen a la mujer reclusa un carácter especial de vulnerabilidad, no solo por su esencia femenina, sino por su rol como madres, esposas, donde sea el espacio de reclusión, más que un agente correctivo, un agente constructivo con resultados positivos en toda la comunidad que la rodea en libertad. En este sentido, los actuales programas transversales, que se articulan con los procesos de resocialización en los establecimientos penitenciarios, deben procurar no solo abrirse a los distintos miembros que conforman las redes de apoyo del condenado, como sus familias, sino que también deben generar estrategias para un mayor acceso a los mismos por parte de la población penitenciaria

- Acceso al derecho a la educación en reclusión como pauta resocializadora: se debe promover bajo la colaboración integral de los diferentes entes del Estado, con una perspectiva de tratamiento transversal desde un punto de vista integral. Lo que hace necesario considerar que la resocialización, como principal función de la pena en el momento de la ejecución penitenciaria, no se satisface solo con la existencia de actividades de trabajo, estudio y enseñanza dentro de los programas institucionales, sino que estas deben apuntar a crear herramientas para la socialización de los condenados y su efectivo regreso e incorporación a sus redes sociales y familiares con posterioridad a la ejecución de la pena.

- Dignidad humana y cárcel: la única manera de dignificar al ser humano en estado de reclusión es la educación, para que, a través del conocimiento, adquiera la libertad de sus propias culpas, conozca y defienda los derechos de todos sus congéneres y sea capaz de participar en una sociedad pacifica, la cual lo conduzca a vivir en libertad como un ser digno de vivir dignamente.

- Reincidencia: la única forma de evitar la reincidencia delictiva y sus consecuencias, es educar desde la casa y la sociedad, y cuando le aplique la cárcel no hay más posibilidades de disminuir la reincidencia. 


\section{Conclusiones}

- La mujer sigue siendo víctima de vulneración de sus derechos sociales, económicos y culturales en la situación de reclusión. Ejemplo de esto se evidencia en el informe de la Defensoría del Pueblo de Boyacá, que indicaba que para las 192 reclusas de la cárcel de Sogamoso existe un solo televisor, mientras que para los hombres hay en promedio un televisor por cada 20 hombres; hay carencia de aulas de clase: mientras que para la población masculina hay tres aulas y el aula biblioteca, para las mujeres hay un salón pequeñísimo. Para los hombres hay además tres talleres de aprendizaje, y para las mujeres no hay ninguno.

- Es urgente crear políticas públicas integradoras en educación en todos los contextos, ya que es la única herramienta liberadora y que permite la participación democrática de todos los ciudadanos, así como el conocimiento y promulgación de los derechos humanos.

- En el ámbito universal, la jurisprudencia converge en el respeto de la dignidad del sujeto a través del goce de todos sus derechos humanos, incluido allí el derecho a la educación sin limitación del contexto donde se aplique.

- En Colombia existe un ordenamiento jurídico sobre el derecho a la educación, aplicable al contexto carcelario, con la funcionalidad de la resocialización de los presos.

- La igualdad de acceso al goce de derechos por parte de hombres y mujeres se aprecia aún con muchas falencias. Como se planteó desde el inicio de este documento, la equidad de género implica la posibilidad de tratamientos diferenciales para corregir desigualdades de base. Las mujeres tienen necesidades específicas, diferentes a los hombres, sus roles en la sociedad han evolucionado de tal manera que reclaman espacios de participación en los diferentes ámbitos de desarrollo.

- Es evidente la crisis carcelaria donde se están vulnerando los derechos humanos de los presos.

- La reincidencia surge como una causa de la ineficacia de los procesos de resocialización.

- Las políticas públicas que plantea el Estado se orientan al tratamiento penitenciario y judicial, y deben examinar la complejidad del problema de hacinamiento y plantear metas a corto, medio y largo plazo. 
- El cumplimiento de la pena debe ir más allá del castigo y generar expectativas de vida encaminadas al proceso de resocialización del sujeto.

- La población carcelaria, en el momento de su ingreso a la cárcel, evidencia un bajo nivel de escolaridad.

- La educación en derechos humanos debe ser un programa transversal en todos los procesos formativos, ya que genera un doble impacto efectivo en el sujeto y en la sociedad.

\section{Referencias}

Azóala, G. \& Yacamán, C. (1997). Las mujeres olvidadas. México: Editorial Publicaciones UAM - Azcapotzalco.

Congreso de la República de Colombia (1993). Ley 65 de 1993. Régimen penitenciario de Colombia.

Corte Constitucional (2002). Sentencia T 881/02. Consideraciones Generales dignidad humana del recluso.

Corte Constitucional (1998). Sentencia T-153/98.

Corte Constitucional (2013). Sentencia T-388/13.

Corte Constitucional (1998). Sentencia de Tutela 153. M. P. Eduardo Cifuentes Muñoz.

Defensoría del pueblo. (2014, 19 de feb.). Informe sobre la situación carcelaria en Colombia. Recuperado de https://www.youtube.com/watch?v=JPZX kSoR4jg

Departamento Nacional de Planeación -DNP- (1995). Conpes 2797 de 1995. Bogotá: DNP.

Departamento Nacional de Planeación -DNP- (2000). Conpes 3086 de 2000. Bogotá: DNP.

Departamento Nacional de Planeación -DNP- (2004). Conpes 3277 de 2004. Bogotá: DNP. 
Departamento Nacional de Planeación -DNP- (2009). Conpes 3575 de 2009. Bogotá: DNP.

Departamento Nacional de Planeación -DNP- (2015). Conpes 3828 de 2015. Bogotá: DNP.

Freire, P. (1965). Pedagogía del oprimido. Buenos Aires: Siglo XXI.

Giacomello, C. (2007). Rompiendo la zona del silencio: testimonios sobre el penal de máxima seguridad del Altiplano, antes La Palma. Bogotá: Dipon.

Inpec (2012). La acción preventiva en materia de Derechos Humanos No. 00001: en cumplimiento jurisprudencial visita íntima. Bogotá: Inpec.

Inpec (2010). Informe de reincidencia 2010-2014. Bogotá: Inpec.

Inpec. (2015, jun.). Informe estadístico. Recuperado de http://www.Inpec.gov.co/ portal/page/portal/Inpec/Institucion/Estad\%EDsticas Informes\%20y\%20 Boletines\%20Estad\%EDsticos

Lagarde, M. (2005). Los cautiverios de las mujeres: madres, esposas, monjas, putas, presas y locas. México: Universidad Nacional Autónoma de México.

Magendzo, A. (2002). Pedagogía crítica y educación en derechos humanos. Chile: www.redacademica.edu.co

Messina, G. (2015). Educación en contexto de encierro como pauta e resocialización. Recuperado de: http://www.pensamientopenal.com.ar/system/files/2015/01/ doctrina36449.pdf

Muñoz, V. (2009). Informe sobre el derecho a la educación de las personas privadas de libertad. Relator Especial de la ONU sobre el derecho a la educación (2004-2010).

Ossa, R. (1998). Criminalidad y criminalización de la mujer en la región andina. Caracas: Nueva Sociedad.

Pacto Internacional de los Derechos Económicos, Sociales y Culturales. (1966). Adoptado por la Asamblea General de las Naciones Unidas el 16 de diciembre de 1966. ONU.

Procuraduría General de la Nación (2006). Mujeres y prisión en Colombia. Recuperado de http://www.procuraduria.gov.co/portal/media/file/descargas/ publicaciones/mujeresyprisionencolombia.pdf 
Scarfó, F. J. (2002). El derecho a la educación en las cárceles como garantía de la educación en derechos humanos. Revista IIDH.

Scarfó, F. \& Aued, V. (2012). El derecho a la educación en las cárceles: abordaje situacional. Aportes para la reflexión sobre la educación como derecho humano en contextos de la cárcel. Argentina: Revista Eletrônica de Educação, Vol. 7, No 1, Universidade Federal de São Carlos, Brasil. ISSN 1982-7199.

Tomasevski, K. (2003). Contenido y vigencia del derecho a la educación. En Cuadernos Pedagógicos, IIDH, San José de Costa Rica. 